Arch. hist. jap. Vol. 14, n. 2 (April 1958).

S. $185-198$.

Anat. Inst. d. Med. Fak., Univ. Okayama (Vorstand: Prof. M. SEKI).

\title{
Über die örtlichen Verschiedenheiten der subepithelialen retikulofibrohistiocytären Gemeischaft der Mundhöhle bei der gesunden Maus.
}

健常な二十日鼠の口腔上皮下の網線組細胞連合の部位的差異と就いて。 Takayasu KITAMURA 北村 貴 保.

(Eingegangen am 16. Dezember 1957.)

Systematische Untersuchungen über die subepitheliale retikulofibrohistiocytäre Gemeinschaft sind neuerdings vielfach angestellt worden. Z. B. liegen die Arbeiten von SEKI (1941, 1953) an der menschlichen Nasenhöhle, die von NAGANUMA (1943) am oberen Luftwege der Maus, die von MATSUI (1953) an der menschlichen Paukenhöhle, die von MIYAMOTO (1953) am Harnwege des Kaninchens, die von YASUDA (1957) an der Kieferhöhle des Kaninchens und die von ONO (1957) am Zahnfleisch der Maus vor.

Der Verfasser vorliegender Mitteilung untersuchte die unter dem Epithel des ganzen Bəreiches der Mundhöhle gelegene retikulofibrohistiocytäre Gemeinschaft der Maus, und zwar mit besonderer Rücksichtigung der örtlichen Verschiedenheiten derselben.

\section{Material und Methode.}

Der Kopf einer normalen erwachsenen Maus wurde nach der Entfernung des Schädeldaches und Gehirns in 10\%iger Formalinlösung fixiert und durch 5\%ige Trichloressigsäurelösung entkalkt. Der Kopf wurde dann in der Medianebene in zwei Hälften getrennt und in Celloidin eingettet. Man schnitt die eine Hälfte in der frontalen und die andere in der sagittalen Richtung in Serienschnitte von $15 \mu$ Dicke. Jeder fünfte Schnitt wurde hintereinander mit Hämatoxylin-Eosin und nach der MÖLLENDORFFschen Eisenhämateinlackmethode gefärbt.

In der unteren Beschreibung sind die Namen der Bindegewebszellen folgendermaßen abgekürzt: Retikulumzelle Rtc, Retikulohistiocyt Rthc, Fibrocyt Fbc, Fibrohistiocyt Fbhc, Histiocyt Hsc, Monocyt Mnc und Lymphocyt Lmc.

\section{Resultate und ihre Auswertung.}

Die Wand der Mundhöhle wurde in acht Abschnitte eingeteilt, d. h. Mundlippe, Wange, Schleimhautteil des Unterkiefers unter dem lingualen Zahnfleisch, Mundboden, Zahnfleisch, harter und weicher Gaumen und Zunge. Die gefundenen Prozentsätze der verschiedenen Zellen in der subepithelialen Bindegewebsschicht in verschiedenen Bezirken der Mundhöhle sind übersichtlich in Tabelle 1 zusammengestellt. Die $L m c$ sind bei der statistischen Untersuchung ausgeschlossen, weil sie an Zahl zu wenig waren. Die Bestimmung der Zellenzahl vollzog sich mit Hilfe des Okular- 
mikrometers, und zwar in 40-80 Sehfeldern.

Tabelle 1.

\begin{tabular}{|c|c|c|c|c|c|}
\hline & \multirow{2}{*}{$\begin{array}{l}\text { Durchschnittszahl } \\
\text { der Bdg.-zellen in } \\
\text { einem Sehfeld }\end{array}$} & \multicolumn{4}{|c|}{ Zellarten $(\%)$} \\
\hline & & $\begin{array}{l}\text { Rtc u. } \\
\text { Fbc }\end{array}$ & $\begin{array}{l}\text { Rthe u. } \\
\text { Fbhe }\end{array}$ & $\mathrm{Hsc}$ & Mnc \\
\hline Mundlippe & 3.2 & 76 & 21 & 2 & 1 \\
\hline Wange & 3.6 & 79 & 18 & 3 & \\
\hline $\begin{array}{l}\text { Unterhalb d. lingua- } \\
\text { len Zahnfleisches d. } \\
\text { Unterkiefers }\end{array}$ & 3.7 & 82 & 18 & & \\
\hline Mundboden & 3.0 & 94 & 6 & & \\
\hline Zahnfeisch & 3.6 & 80 & 13 & 5 & 2 \\
\hline Harter Gaumen & 3.9 & 83 & 13 & 3 & 1 \\
\hline Weicher Gaumen & 4.0 & 75 & 20 & 2 & 3 \\
\hline $\begin{array}{l}\text { Zungenrücken u. } \\
\text { Zungenrand }\end{array}$ & 3.8 & 86 & 14 & & \\
\hline $\begin{array}{l}\text { Untere Fläche d. } \\
\text { Zunge }\end{array}$ & 3.4 & 92 & 8 & & \\
\hline
\end{tabular}

Um die jeweilige komplizierte Zusammensetzung aus den verschiedenen Zellen anschaulich zu machen, wurden die folgenden Abkürzungsformen gebraucht. Der RF-Typus (retikulofibrocytärer Typus) ist der, in welchem die Rtc und F'bc 7080\%ig oder einen noch größeren Anteil der ganzen Zellen ausmachen; im RFHTypus (retikulofibrohistiocytären Typus) sind die Rtc, Fbc und Hsc am zahlreichsten, ihre Zahl beträgt 50-60\%; im H-Typus (histiocytären Typus) finden sich die Hsc zu mehr als 30\%. Über anderweitige Typen, den MH-Typus (monohistiocytären Typus), den M-Typus (monocytären Typus), den Atr-Typus (atrophischen Typus) usw. sehe man MATSUI (1953).

\section{Mundlippe.}

Die Bindegewebsschicht zwischen dem Epithel und der Muskelschicht ist 15$50 \mu$ dick, und die bindegewebigen Papillen sind kaum gebildet. Dicht unter dem Epithel sind $F b c$ und $F b h c$ zahlreich vorhanden, aber auch $H s c$ werden dazwischen bemerkt. Die Rtc sind kaum zu finden. Das Zahlenverhältnis der Frbc, Fbhc und Hsc ist 15:8:1, und das Gewebsbild gehört zum RFH-Typus (Abb. 2). Die Bindegewebsfasern sind schwach entwickelt.

In der tieferen Schicht laufen die Bindegewebsfasern dicht nebeneinander und bilden eine straffe Schicht. Die zwischen den Fasern vorhandenen $F b c$ sind demnach abgeplattet. Die $F b c, F b h c$ und $M n c$ finden sich im Verhältnis von $46: 3: 1$. Das Bild ist der RF-Typus.

\section{Wange.}

Es läßt sich die Wangenschleimhaut vorteilhafterweise in drei Abschnitte einteilen. Die vom Mundwinkel bis zu der dem medialen Rand des ersten Molarzahns entgegenliegenden Stelle wird als A-Teil benannt. Die hintere Abteilung der Wangenschleimhaut wird in zwei eingeteilt, $d . h$. B- und C-Teil je ober- und unterhalb 


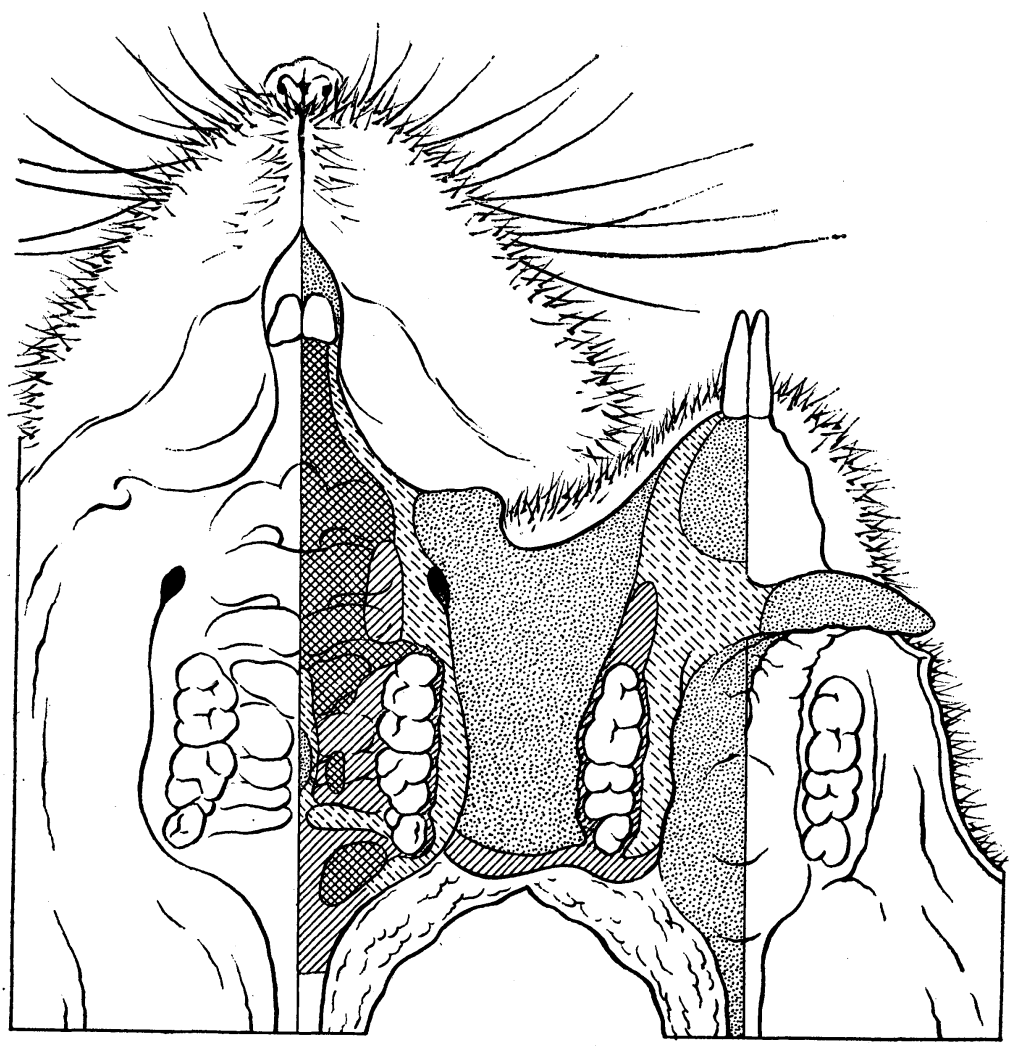

Abb. 1. Landkarte der Mundhöhlenschleimhaut einer Maus bezüglich der Dicke der subepithelialen Bindegewebsschicht.

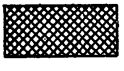
mehr als $150 \mu$,

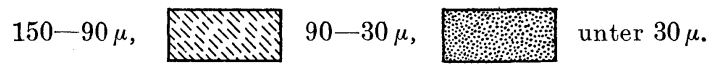

der horizontal liegenden Buccalraphe des Epithels.

Die Bindegewebsschicht im A- und C-Teil ist größtenteils 25-60 $\mu$ dick, im A-Teil aber stellenweise $80-95 \mu$. Im B-Teil ist sie nur 15-30 $\mu$ dick. 1m A-Teil sind die Papillen schwach entwickelt, so daß die Grenze gegen das Epithel fast flach ist. Sie sind jedoch im B-Teil gut entwickelt. Der C-Teil hat wieder schlecht entwickelte Papillen.

Im A-Teil enthält das subepitheliale Gewebe, wie in Abb. 3 gezeigt ist, Bindegewebsfasern nur gering, aber zahlreiche $R t c$ und $F b c$ und auch ihre Übergangsformen zu Hsc. Die Rtc oder Fbc, Rthc oder Fbhc und Hsc sind im Verhältnis von $10: 2: 1$ vorhanden.

Das Bild des subepithelialen Gewebes im B- und C-Teil ist fast das gleiche. In den Papillen befinden sich Rthc und Fbhc nicht wenig an Zahl (Abb. 4). Die Rtc oder $F b c$, Rthc oder $F b h c$ und Hsc ist in $24: 8: 1:$ das Bild ist der RFH-Typus. In der Tiefe ist das Gewebe reich an Fbc, und das Bild ist vom RF-Typus. Kleine 
Hsc werden im C-Teil mehr getroffen als im B-Teil.

\section{Unterhalb des lingualen Zahnfleishes des Unterkiefers.}

Die Dicke der Bindegewebsschicht beträgt hier $40-80 \mu$. Sie ist dicker in der Nähe vom Zahnfleisch, und von diesem entfernt wird sie dünner. Die Papillen sind sehr gut entwickelt. Die in ihnen vorhandenen Bindegewebszellen sind Rtc und $R$ thc im Verhältnis von $2: 1$ (Abb. 5). An der Basis der Papillen werden hauptsächlich $F b c$ gesehen. Die Bindegewebsfasern sind in den Papillen natürlicherweise sehr spärlich. Sie finden sich auch in den sonstigen Stellen nur in geringem Maße. Das Gewebsbild ist der RFH-Typus.

\section{Mundboden.}

Die Bindegewebsscicht ist von 13-25 $\mu$ Dicke. Papillen gibt es kaum, und die Grenze zwischen dem Bindegewebe und dem Epithel ist flach. Die Bindegewebsschicht ist faserreich, und die dort vorhandenen Zellen sind meistens kleine $F b c$ (Abb. 6). Hsc und $M n c$ sind kaum zu finden. Diese finden sich im Verhältnis von $16: 1$, und das Gewebsbild gehört zum Atr-Typus.

\section{Zahnfleisch.}

Die $F b c$ und Rthc stellen den Mutterboden des subepithelialen Bindegewebes dar, aber dem Zahnhals entlang gibt es, wie in Abb. 7 gezeigt ist, neben den genannten Zellen zahlreiche $R t h c$ und $F b h c$, aber auch zerstreut $H s c$ von kleiner Form nnd Mnc. Das Verhältnis der Hsc zu Mnc ist $3: 1$. Das Gewebsbild ist der H-Typus. Die Bindegewebsfasern sind im allgemeinen schwach entwickelt. Dem $L m c$ ähnliche Zellen werden angetroffen.

\section{Harter Gaumen.}

Hinter den oberen Schneidezähnen liegen drei (erste, zweite und dritte) querlaufende Gaumenfalten. Die Bindegewebsschicht ist in der ersten Gaumenfalte am dicksten und ist in der zweiten und dritten Falte immer dünner. Die Dicke beträgt an der dicksten Stelle der ersten Falte $650 \mu$, und in der Mitte derselben findet sich ein Knorpel (Abb. 8). Dagegen haben andere Falten keinen solchen. In der zweiten Gaumefalte ist die Bindegewebsschicht $500 \mu$ und in der dritten $260 \mu$ dick. Hinter der dritten Gaumenfalte folgen auf jeder Seite fünf kleinere Querfalten. Die Bindegewebsschicht ist hier in der Mediangegend am dünsten $(39-65 \mu)$ und ist lateralwärts nach den Molarzähnen dicker $(176-385 \mu)$. Die Papillen werden nur in der Nähe der Zähne gesehen, und ihre Begrezung gegen das Epithel ist zum größten Teil flach.

Dicht unterhalb des Epithels finden sich zahlreich $R t c$, Rthc und Fbhc (Abb. 9). Die Rtc oder Fbc, Rthc oder Fbhc, Hsc und Mnc finden sich in $79: 16: 4: 1$. Aber in den Schleimhauteilen, in welchen die bindegewebige Schicht verhältnismäßig dünner ist, sind die Bindegewebszellen in der Regel locker vorhanden (Abb. 10), uud dicht unter dem Epithel werden die $F b c$ und $F b h c$ im Verhältnis von $8: 1$ gefunden. In der tieferen Stelle kommen $F b c$ zahlreich vor. Nahe dem Knochengewebe findet sich gefäßreiches lockeres Gewebe (Abb. 9). 


\section{Weicher Gaumen.}

Die Dicke der Bindegewebsschicht ist je nach der Entwicklung der Gaumendrüsen sehr verschieden, beträgt aber gewöhnlich $90-120 \mu$.

Subepithelial gibt es $R t c$ und auch aus ihnen entstandene Fbc und Fbhc. Die bindegewebigen Papillen, in denen sich zahlreiche $R t h c$ und Fbhc finden, sind relativ gut entwickelt, und ihr Gewebsbild ist der RFH-typus (Abb. 11). Man sieht auch zerstreut kleine Hsc. Die Bindegewebsfasern kommen nur in geringem Maße vor.

\section{Zunge.}

Die Bindegewebsschicht ist am Zungenrücken dick, am Zungenrand dünn und auf der Unterfläche der Zunge noch dünner. Sie ist am Rücken und am Rand der Zunge $26-40 \mu$ dick und auf ihrer Unterfläche $20-28 \mu$.

Am Zungenrücken sowie -rand finden sich die Rtc dicht unter dem Epithel zahlreich (Abb. 12), uud zwar sind die Rtc oder Fbc zu den Rthc oder Fbhc im Verhältnis von $6: 1$ vorhanden. Das Gewebe ist vom RF-Typus. Auf der Unterfläche der Zunge werden unter dem Epithel fast keine Rtc gesehen. Hier gibt es reichlich Fasern, und die meisten Zellen sind abgeplattete Fbc (Abb. 13).

\section{Zusammenfassung.}

Zur Untersuchung wurde eine ganz normal erscheinende, erwachsene Maus benutzt.

1. Die Reihenfolge der Dicke der subepithelialen Bindegewebsschicht der Mundhöhle ist: im größten Teil des harten Gaumens > am weichen Gaumen > am Zahnfleisch $>$ an den Lippen $>$ am unter dem lingualen Zahnfleisch gelegenen Schleimhautteil des Unterkiefers $>$ in der vorderen Hälfte der Wangenschleimhaut $>$ am Rücken und Rand der Zunge $>$ in der hinteren Hälfte der Wangenschleimhaut $>$ auf der Unterfläche der Zunge $>$ am Mundboden.

2. An dem harten Gaumen, Zahnfleisch, weichen Gaumen, Zungenrücken und auf der lateralen Fläche der Zunge sind unter dem Epithel hauptsächlich Retikulumzellen und Fibrocyten vorhanden. An den Mundlippen, Wangen und unterhalb des lingualen Zahnfleisches des Unterkiefers gesellen sich nicht wenige Retikulo- und Fibrohistiocyten noch dazu. In der Tiefe der Schleimhaut treten zahlreiche Fibrocyten mit den Bindegewebsfasern auf.

3. Die Retikulohistiocyten und Fibrohistiocyten kommen der Zahl nach in der folgenden Reihenfolge vor: Mundlippe $>$ weicher Gaumen $>$ Wangen $>$ unterhalb des lingualen Zahnfleisches des Unterkiefers $>$ Zahnfleisch $>$ harter Gaumen $>$ untere Fläche der Zunge $>$ Mundboden.

4. Diejenigen Orte, an welchen die Histiocyten im subepithelialen Gewebe mehr oder weniger zahlreich zu sehen sind, sind das Zahnfleisch, der harte und weiche Gaumen, die Wange und die Mundlippe, dagegen finden sie sich unterhalb des Zahnfleisches des Unterkiefers, an der unteren Fläche der Zunge und am Mundboden unter dem Epithel selten. Das subepitheliale Gewebe der letzteren zwei Orte ist mit reichlichen Bindegewebsfasern versehen, und die dort befindlichen Bindegewebszellen sind allermeist Fibrocyten. 
5. Kurz zusammenfassend kann man sagen, daß die unter dem dicken, verhornten Epithel vorhandenen Zellen meistens Retikulumzellen und Fibrocyten sind, daß aber unter dem mittelmäßig dicken, nicht verhornten Epithel sowie in den gut entwickelten bindegewebigen Papillen auch Retikulohistiocyten, Fibrohistiocyten und Histiocyten vorkommen, ausgenommen, daß die Histiocyten sich unterhalb des Zahnfleisches des Unterkiefers selten zu sehen sind. Unter dem dünnsten Epithel sind die Bindegewebszellen dünn und schlank.

\section{内 容自抄.}

健常な成長二十日鼠の口腔壁の連続切片を鉄へマテインラック法とへマトキシ リンとエオンンで染め, 上皮下の結織層の厚さとそこの網線組細胞連合の状態を 調ベた.

口腔上皮下組織の厚さの順位は, 硬口蓋の大部分 $>$ 軟口蓋 $>$ 歯肉 $>$ 口唇 $>$ 下顎 の舌側歯肉の下方の粘膜 $>$ 煩の前半 $>$ 舌背と舌側面 $>$ 煩の後半 $>$ 舌下面 $>$ 口腔底 である。

口腔粘膜の大部分では上皮下の結織層の細胞は細網細胞と線維細胞が主で，そ れにとの両種の細胞より派生したと思える網組球と線組球が多少加わり，な招所 によっては小形の組織球が散在する。

表面が争化した厚い上皮の下飞存在する細胞は多くは細網細胞と線維細胞であ る。厚さが中等度で，表面が售化しない上皮とよく発達した結織性乳頭には網組 球，線組球が比較的多い，但し組織球は下顎の舌側㐘肉の下方の粘膜だけには稀 にしか見られない。上皮の最も薄いところである舌下面と口腔底の上皮下の細胞 は, 線維細胞が主であり, その形薄弱で, 数も少ない。

\section{Literatur.}

Kitamura, T.: Die örtlichen Verschiedenheiten der epithelialen Struktur der Mundhöhle bei der Maus. Arch. hist. jap. 12 (1957). - Matsui, K.: Histometrische Studien über die Bindegewebszellen in der Schleimhaut der Paukenhöhle und ihrer Nebenhöhlen in der Fetalzeit beim Erwachsenen. Arch. hist. jap. 4 (1953 a). - Die Veränderungen des Epithels und der Bindegewebszellen an der mit Formalin gereizten Paukenschleimhaut der Maus. Arch. hist. jap. 4 (1953 b). - Miyamoto, J.: Über das fibrohistiocytäre System der Schleimhaut des Harnwegs des gesunden Kaninchens. Arch. hist. jap. 5 (1953). - Naganuma, K.: Das Retikulum und das fibrohistiocyäre System in der Schleimhaut des oberen Luftweges der gesunden Maus. (Jap.) Kaibo Z. 21 (1943). - Ono, H.: Untersuchung der Veränderung der gereizten Bindegewebszellen unter dem Ansatz des Epithels am Zahn bei der Maus. Arch. hist. jap. 12 (1957). - Seki, M.: Retikulumzellen und ihre Abkömmlinge in der Nasenschleimhaut des Menschen. Z. Zellforsch. 31 (1941); Arch. hist. jap. 4 (1953).

- Yasuda, K.: Über die retikulofibrohistiocytäre Gemeinschaft der Kieferhöhlenschleimhaut des Kaninchens und ihre Antikörperproduktion, nebst einem Versuch über die Durchläßigkeit des Schleimhautepithels für Farbstoffe. Arch. hist. jap. 11 (1957). 
Tafelabbildungen. 


\section{Erläuterung zu den Tafelabbildungen.}

$C h$ Knorpel, $D r$ Drüse, $E p$ Epithel, Fbc Fibrocyt, Fbhc Fibrohistiocyt, Hsc Histiocyt, M Muskelfasern, Mnc Monocyt, Rtc Retikulumzelle, Rthc Retikulohistiocyt, S Schmelzsubstanz ist infolge der Entkalkung ausgelöst.

Abb. 2. Aus der unteren Lippe. Sagittaler Schnitt. Man sieht dicht unter dem Epithel die $F b c, F b h c$ und $H s c$. In der tieferen Schicht sind die Bindegewebsfasern dicht vorhanden. $400 \times$

Abb. 3. Aus dem Vorderteil der Wangenschleimhaut. Frontalschnitt. Spärliche Bindegewebsfasern. Man sieht die Rtc und Fbc und ihre Übergangsformen zu Hsc. $400 \times$

Abb. 4. Aus der den Molarzähnen entgegenliegenden Stelle der Wange. Frontaler Schnitt. In dem Papillen befinden sich Rthc und Fbhc. $400 \times$

Abb. 5. Aus der Schleimhaut unterhalb des lingualen Zahnfleisches des Unterkiefers. Frontaler Schnitt. $F b c$ und $F b h c$ in den Papillen. In dem Basalteil der Papillen aber hauptsächlich Fbc. $400 \times$ 
T. KITAMURA (1)
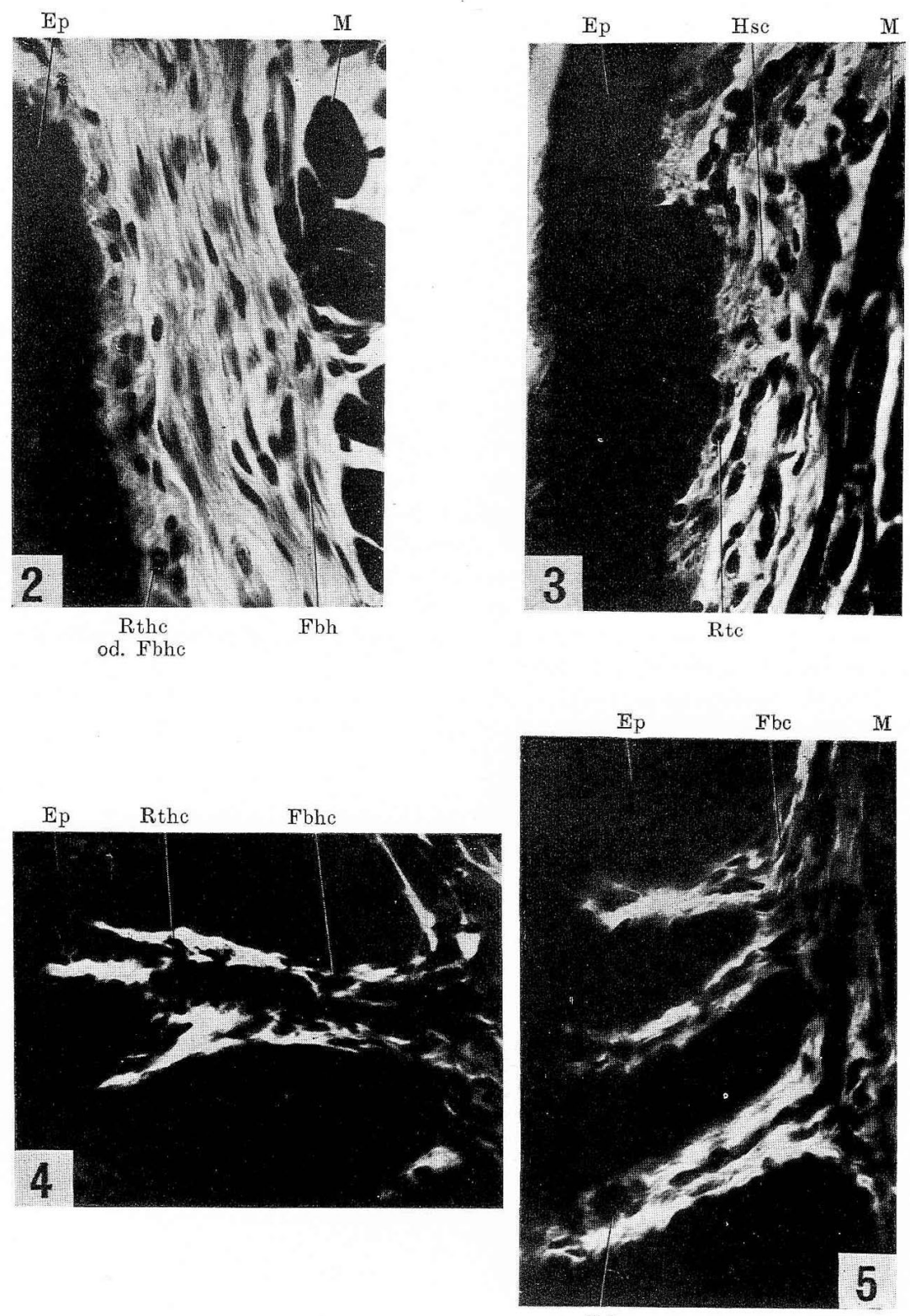

Rthc 
Abb. 6. Aus dem Mundboden. Frontaler Schnitt. Die Bindegewebsfasern sind reichlich vorhanden. Die Zellen sind meistens kleine Fbc. $400 \times$

Abb. 7. Aus dem Zahnfleisch. Frontaler Schnitt. Die Mehrzahl der Zellen ist Rtc und $F^{\prime} b c$. Kleine $H s c$ oder Mnc werden angetroffen. $4.00 \times$

Abb. 8. Aus der Schleimhaut der hinter den oberen Schneidezähnen liegenden ersten Gaumenfalte. Frontaler Schnitt. $100 \times$

Abb. 9. Aus dem harten Gaumen. Frontaler Schnitt. Dicht unter dem Epithel finden sich $R t h c$ und Fbhc, in der tieferen Schicht aber fast lediglich $F b c .400 \times$ 
T. KITAMURA (2)

Fbc

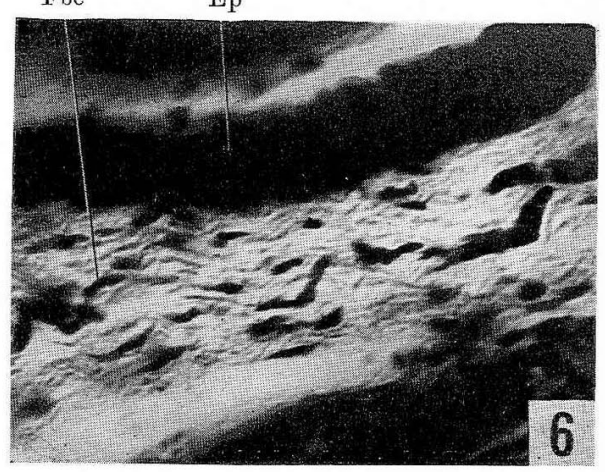

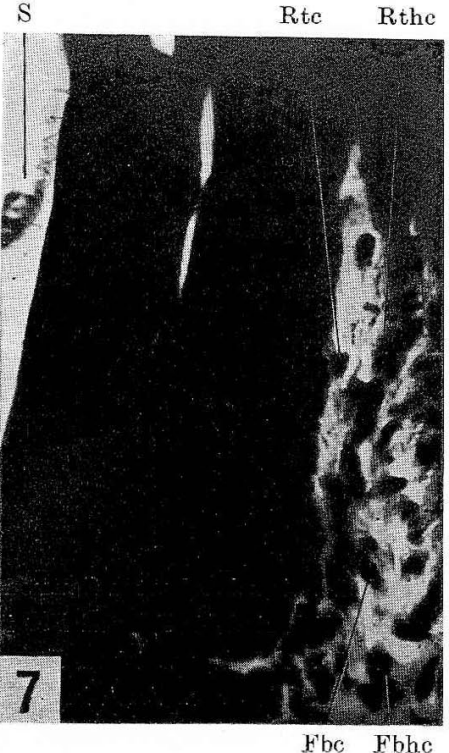

Hsc Rtc Ep Rthc

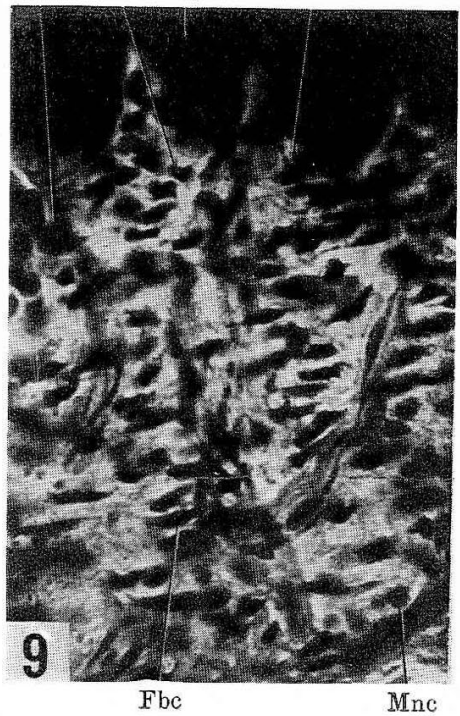


Abb. 10. Aus dem harten Gaumen mit dünneren Bindegewebsschicht. Frontaler Schnitt. Hauptsächlich sind die Rtc und Fbc im Bindegewebe zu sehen. $400 \times$

Abb. 11. Aus dem weichen Gaumen. Frontaler Schnitt. Unter dem Epithel kommen die Fbhc und Rthc vor. Auch keine Formen der Hsc sind zu treffen. $400 \times$

Abb. 12. Aus dem Zungenrand. Frontaler Schnitt. Zahlreiche Rtc und $F b c$ in der Nähe des Epithels. $400 \times$

Abb. 13. Aus der Unterfläche der Zunge. Frontaler Schnitt. Wenige $F b c$ zwischen den Bindegewebsfasern. $400 \times$ 
T. KITAMURA (3)
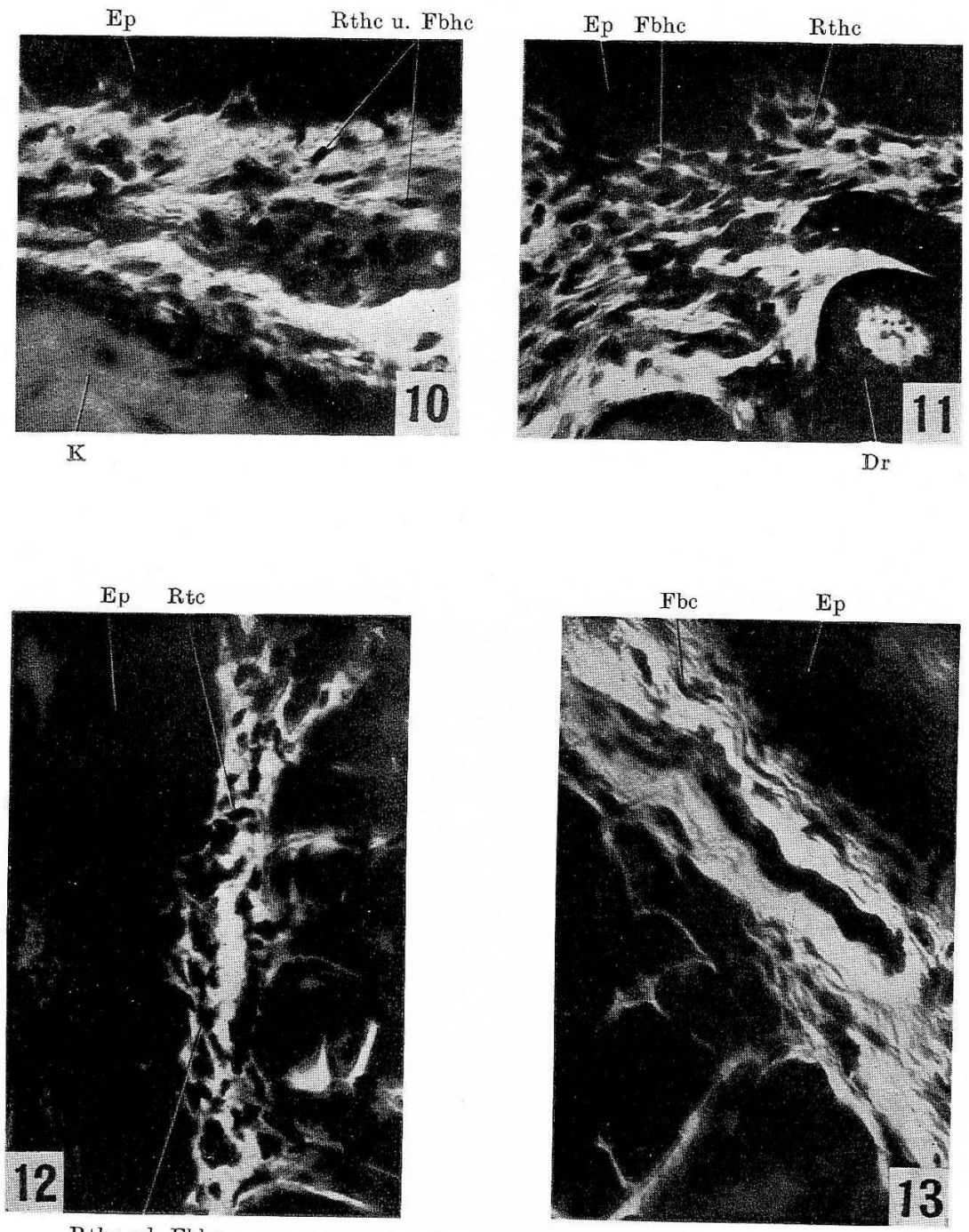

Rthe od. Fbhe 